\title{
La coevaluación del equipo versus el resultado académico
}

\section{Jose Domènech i de Soria ${ }^{a}$, Elena De la Poza Plazab, Maria Cinta Vincent Vela ${ }^{c}$, Jaime} Lloret Mauríd

a,b,c,d Universitat Politècnica de València; ajdomenech@upvnet.upv.es; belpopla@esp.upv.es; mavinve@iqn.upv.es; d jlloret@dcom.upv.es

\begin{abstract}
This paper deals with the implementation of a system of peer assessment of collaborative work as a tool to measure students level of awareness of the work done in groups. Then, their collaborative work perception is compared with the academic results obtained by the group.

The teacher provides a rubric for assessing the ability of collaborative group work. This group reflection on the methods and skills developed by working as a unit encourages critical analysis of individuals.

The application of this methodology intends to provide information to the group, and teachers, to better understand the value that the group makes about its capabilities and compare those with their academic results.

Keywords : collaborative work, peer assessment, academic results , critical analysis.
\end{abstract}

\section{Resumen}

En este trabajo se describe la implementación de un sistema de coevaluación del trabajo colaborativo de los alumnos como herramienta para medir el nivel de conciencia que los alumnos adquieren del trabajo desarrollado grupalmente, y comparar su percepción con los resultados académicos obtenidos por el grupo.

El docente proporciona una rúbrica de evaluación de la capacidad de trabajo colaborativo del grupo. Con ello se fomenta la reflexión grupal sobre los procedimientos y aptitudes desarrollados en el ejercicio del trabajo colaborativo, y así fomentar el análisis crítico de los individuos como una unidad de trabajo.

A través de esta metodología se pretende aportar información al grupo, y al docente, para conocer mejor la valoración que el grupo hace sobre sus capacidades y compararlos con los resultados académicos obtenidos. 
La coevaluación del equipo versus el resultado académico

Palabras clave: trabajo colaborativo, coevaluación, resultados académicos, análisis crítico.

\section{Introducción}

Entre las competencias genéricas que el estudiante de educación superior debe mostrar se encuentra la habilidad para trabajar en equipo (Brown y Knight, 2012). Así, la práctica del trabajo en equipo durante la etapa universitaria pretende fomentar la capacidad del individuo para alcanzar un fin común, tomar iniciativa, gestionar conflictos y recursos, contribuir al trabajo en común, sintiéndose responsable y partícipe del mismo.

Tradicionalmente la evaluación docente del trabajo en equipo o colaborativo se ha basado en actos o pruebas tales como presentaciones públicas del proyecto o trabajo realizado, o productos como son los documentos en soporte papel o digital del trabajo realizado, todos ellos ponderados en el resultado académico de la asignatura.

Sin embargo, estos sistemas de evaluación, si bien son útiles para conocer el grado de dominio de la materia, así como para valorar la capacidad de comunicación verbal y escrita del grupo, no aportan información relativa a la percepción que el alumno y el grupo tienen sobre sus habilidades para trabajar de forma colaborativa y por tanto sobre la capacidad de trabajar en equipo.

El alumno de forma individual así como el equipo necesitan evaluar y por tanto ser conscientes de las dimensiones que componen el trabajo colaborativo, y ser críticos con la actuación grupal. El docente debe proporcionar las herramientas para que el equipo reflexione sobre su desarrollo y evolución, con el fin de poder establecer medidas correctoras de mejora o para reforzar actitudes positivas del grupo.

En este contexto se analiza para la asignatura de Empresa del grupo de alto rendimiento académico (ARA) impartida en el Grado de Ingeniería Aeroespacial la coevaluación del trabajo colaborativo tanto de forma grupal como individual.

\section{Objetivo}

El objetivo del trabajo es medir el desarrollo de las competencias de trabajo en equipo de los alumnos, y contrastar la hipótesis de si el resultado de los trabajos colaborativos, que viene dado por las calificaciones académicas obtenidas por los alumnos de forma grupal (trabajo escrito, y presentación oral) implican mejores competencias de trabajo colaborativo (Wilson, 2002). Para ello, se utiliza la coevaluación (Chen, 2010; Falchicov, 1995; Logan, 2009; Ryley, 1995; Topping, 1998) de forma grupal y pública entre los miembros del equipo, y posteriormente cada componente del grupo evalúa las competencias del trabajo colaborativo de sus compañeros.

\section{Asignatura de Empresa}


La asignatura de Empresa, se imparte en el primer curso del Grado de Ingeniería Aeroespacial en la Universitat Politècnica de València. Se trata de una asignatura básica en la que se analiza la empresa teniendo en cuenta 5 sistemas esenciales : los mercados en los que actúa la empresa y agentes que intervienen, el sistema de producción, el sistema de marketing de la empresa, así como el sistema de inversión y financiación.

En el curso académico 2014/2015 se ha evaluado el proceso de aprendizaje de la asignatura a través de 4 sistemas de evaluación, que se detallan a continuación:

- La evaluación mediante pruebas objetivas (3 tests) a través de tests temporizados de opción múltiple con un peso del 50\% en la nota final de la asignatura. Los contenidos son acumulativos. El estudiante tiene que realizar 3 pruebas a lo largo del semestre a través de la plataforma PoliformaT de la UPV. El primer test tiene un peso del 5\%, el segundo test un peso del $15 \%$ y el tercer test un peso del $30 \%$

- La evaluación mediante trabajo colaborativo tendrá un peso del 30\% en la nota final de la asignatura. Se compondrá de una presentación oral (15\%) y la presentación por escrito de del trabajo de investigación en una área en concreto del sector aeronáutico que comprende el conjunto de tareas realizadas siguiendo una guía proporcionada por el profesor.

-La evaluación mediante observación (prácticas laboratorio) tiene un peso del 10\% en la nota final de la asignatura.

-La evaluación mediante ejercicios propuestos en el aula, con una ponderación del 10\% en la nota final de la asignatura.

\section{El trabajo colaborativo}

Como se ha explicado en la sección anterior, la evaluación del trabajo colaborativo se produce de forma puntual, a través de la comunicación oral de los aspectos más relevantes del trabajo desarrollado por el grupo, y a través del trabajo escrito. Ambas evaluaciones se producen al final del semestre durante las semanas 12-13, cuando se han explicado los contenidos de la asignatura en su totalidad.

Sin embargo, a diferencia del trabajo autónomo del estudiante, el trabajo colaborativo abarca distintas dimensiones, tales como las expectativas que cada uno de los componentes del grupo tiene del trabajo a desarrollar, y del grupo del que forman parte, las habilidades que cada uno de los miembros aporta, las experiencias previas de trabajo en grupo que cada miembro haya tenido, el estado emocional de cada componente del grupo, etc.

De hecho, la constitución de un equipo comporta una de las fases iniciales relevantes del trabajo en grupo. Así, una vez los alumnos conocen los sistemas de evaluación, y el docente les explica la importancia del proceso de constitución del grupo como factor dinamizador del aprendizaje y éxito del trabajo colaborativo, los alumnos de forma individual reflexionan sobre 5 preguntas de respuesta abierta (Tabla 1); tras contestar a dichas preguntas se realizan una serie de actividades en el aula durante tres sesiones consecutivas, 
que hacen interactuar a los alumnos en grupos distintos con la finalidad de estos se conozcan entre sí, y así pudieran dar respuesta a las preguntas formuladas en las cuestiones 4 y 5 (Tabla 1). Transcurridas tres semanas desde el comienzo del curso los alumnos

eligieron a sus compañeros de grupo, y constituyeron sus equipos.

Tabla 1. Preguntas sobre trabajo colaborativo realizadas al inicio del semestre

\author{
1. Have you ever worked by teams? \\ 2. What do you value the most (3 drivers) when working by teams? \\ 3. What do you expect from the collaborative work/project to be developed during this semester? \\ 4. What attitudes/competencies are you looking for in you members' team? \\ 5. In order to build your team, what is the information you need to know about your peers to recruit \\ them and achieve your goals?
}

Fuente: Elaboración propia

Desde la 4 semana a la semana 12 del semestre, los alumnos desarrollaron de forma semanal durante la sesión de aula actividades de forma colaborativa, algunas tareas propuestas que requerían la entrega de un informe o resultados al finalizar la sesión, fomentando así las distintas dimensiones del trabajo en equipo (Tabla 2).

Durante las semanas 12 y 13 los alumnos realizaron las presentaciones públicas ante el resto de sus compañeros y entregaron los documentos escritos. Durante la semana 14, y previo a conocer las evaluaciones que el docente había hecho de las presentaciones orales y de los trabajos escritos de cada equipo, cada grupo realizó una evaluación conjunta de su trabajo en el aula, valorando de 1 a 4 los distintos ámbitos del trabajo colaborativo, tales como la capacidad de trabajo con otras personas, el logro del objetivo, la actitud, la gestión del tiempo, la calidad del trabajo producido, la contribución de cada miembro del grupo al trabajo en común, y la capacidad para resolver problemas de los miembros del grupo.

Asimismo, la rúbrica para la evaluación del trabajo colaborativo se depositó en el Poliformat, para que cada alumno realizara una evaluación personal y privada de cada uno de sus compañeros de grupo. Las coevaluaciones del trabajo colaborativo generadas por cada alumno fueron depositadas en el espacio compartido del Poliformat, al cual tan solo el profesor y el alumno tienen acceso, fomentando la privacidad y libertad de la opinión personal. 
Jose Domènech i de Soria, Elena De la Poza Plaza, Maria Cinta Vincent Vela, Jaime Lloret Maurí

Tabla 2. Rúbrica para la evaluación del trabajo colaborativo

\begin{tabular}{|c|c|c|c|c|}
\hline CATEGORY & 4 & 3 & 2 & 1 \\
\hline $\begin{array}{l}\text { Working with } \\
\text { Others }\end{array}$ & $\begin{array}{l}\text { Almost always listens } \\
\text { to and supports the } \\
\text { efforts of others. Tries } \\
\text { to keep people } \\
\text { working well together. }\end{array}$ & $\begin{array}{l}\text { Usually listens to, } \\
\text { and supports the } \\
\text { efforts of others. } \\
\text { Does not cause } \\
\text { "waves" in the } \\
\text { group. }\end{array}$ & $\begin{array}{l}\text { Often listens to, and } \\
\text { supports the efforts } \\
\text { of others, but } \\
\text { sometimes is not a } \\
\text { good team member. }\end{array}$ & $\begin{array}{l}\text { Rarely listens to, and } \\
\text { supports the efforts } \\
\text { of others. Often is not } \\
\text { a good team player. }\end{array}$ \\
\hline $\begin{array}{l}\text { Focus on the } \\
\text { task }\end{array}$ & $\begin{array}{l}\text { Consistently stays } \\
\text { focused on the task } \\
\text { and what needs to be } \\
\text { done. Very self- } \\
\text { directed. }\end{array}$ & $\begin{array}{l}\text { Focuses on the } \\
\text { task and what } \\
\text { needs to be done } \\
\text { most of the time. } \\
\text { Other group } \\
\text { members can } \\
\text { count on this } \\
\text { person. }\end{array}$ & $\begin{array}{l}\text { Focuses on the task } \\
\text { and what needs to } \\
\text { be done some of the } \\
\text { time. Other group } \\
\text { members must } \\
\text { remind to keep this } \\
\text { person on-task. }\end{array}$ & $\begin{array}{l}\text { Rarely focuses on the } \\
\text { task and what needs } \\
\text { to be done. Lets } \\
\text { others do the work. }\end{array}$ \\
\hline Attitude & $\begin{array}{l}\text { Never is publicly } \\
\text { critical of the project } \\
\text { or the work of others. } \\
\text { Always has a positive } \\
\text { attitude about the } \\
\text { task(s). }\end{array}$ & $\begin{array}{l}\text { Rarely is publicly } \\
\text { critical of the } \\
\text { project or the } \\
\text { work of others. } \\
\text { Often has a } \\
\text { positive attitude } \\
\text { about the task(s). }\end{array}$ & $\begin{array}{l}\text { Occasionally is } \\
\text { publicly critical of } \\
\text { the project or the } \\
\text { work of others. } \\
\text { Usually has a } \\
\text { positive attitude } \\
\text { about the task(s). }\end{array}$ & $\begin{array}{l}\text { Often is publicly } \\
\text { critical of the project } \\
\text { or the work of others } \\
\text { Often has a negative } \\
\text { attitude about the } \\
\text { task(s). }\end{array}$ \\
\hline $\begin{array}{l}\text { Time- } \\
\text { management }\end{array}$ & $\begin{array}{l}\text { Routinely uses time } \\
\text { well throughout the } \\
\text { project to ensure things } \\
\text { get done on time. } \\
\text { Group does not have to } \\
\text { adjust deadlines or } \\
\text { work responsibilities } \\
\text { because of this } \\
\text { person's } \\
\text { procrastination. }\end{array}$ & $\begin{array}{l}\text { Usually uses time } \\
\text { well throughout } \\
\text { the project, but } \\
\text { may have } \\
\text { procrastinated on } \\
\text { one thing. }\end{array}$ & $\begin{array}{l}\text { Tends to } \\
\text { procrastinate, but } \\
\text { always gets things } \\
\text { done by the } \\
\text { deadlines.. }\end{array}$ & $\begin{array}{l}\text { Rarely gets things } \\
\text { done by the deadlines } \\
\text { and group has to } \\
\text { adjust deadlines or } \\
\text { work responsibilities } \\
\text { because of this } \\
\text { person's inadequate } \\
\text { time management. }\end{array}$ \\
\hline $\begin{array}{l}\text { Quality } \\
\text { Work }\end{array}$ & $\begin{array}{l}\text { Provides work of the } \\
\text { highest quality. }\end{array}$ & $\begin{array}{l}\text { Provides high } \\
\text { quality work. }\end{array}$ & $\begin{array}{l}\text { Provides work that } \\
\text { occasionally needs } \\
\text { to be } \\
\text { checked/redone by } \\
\text { other group } \\
\text { members to ensure } \\
\text { quality. }\end{array}$ & $\begin{array}{l}\text { Provides work that } \\
\text { usually needs to be } \\
\text { checked/redone by } \\
\text { others to ensure } \\
\text { quality. }\end{array}$ \\
\hline
\end{tabular}

(c)) EY-NC-ND 2015, Universitat Politècnica de València

Congreso In-Red (2015) 


\begin{tabular}{|c|c|c|c|c|}
\hline Contributions & $\begin{array}{l}\text { Routinely provides } \\
\text { useful ideas when } \\
\text { participating in the } \\
\text { group and in classroom } \\
\text { discussion. A definite } \\
\text { leader who contributes } \\
\text { a lot of effort. }\end{array}$ & $\begin{array}{l}\text { Usually provides } \\
\text { useful ideas when } \\
\text { participating in } \\
\text { the group and in } \\
\text { classroom } \\
\text { discussion. A } \\
\text { strong group } \\
\text { member }\end{array}$ & $\begin{array}{l}\text { Sometimes provides } \\
\text { useful ideas when } \\
\text { participating in the } \\
\text { group and in } \\
\text { classroom } \\
\text { discussion. A } \\
\text { satisfactory group } \\
\text { member }\end{array}$ & $\begin{array}{l}\text { Rarely provides } \\
\text { useful ideas when } \\
\text { participating in the } \\
\text { group and in } \\
\text { classroom discussion. } \\
\text { May refuse to } \\
\text { participate. }\end{array}$ \\
\hline $\begin{array}{l}\text { Problem- } \\
\text { solving }\end{array}$ & $\begin{array}{l}\text { Actively looks for and } \\
\text { suggests solutions to } \\
\text { problems. }\end{array}$ & $\begin{array}{l}\text { Refines solutions } \\
\text { suggested by } \\
\text { others. }\end{array}$ & $\begin{array}{l}\text { Does not suggest or } \\
\text { refine solutions, but } \\
\text { is willing to try out } \\
\text { solutions suggested } \\
\text { by others. }\end{array}$ & $\begin{array}{l}\text { Does not try to solve } \\
\text { problems or help } \\
\text { others solve } \\
\text { problems. Lets others } \\
\text { do the work. }\end{array}$ \\
\hline
\end{tabular}

Fuente: Lucas (2014)

\section{Resultados}

Los 50 alumnos del grupo ARA durante el curso académico 2014/2015, constituyeron 11 equipos.

De los resultados obtenidos de las 5 preguntas iniciales realizadas a los alumnos al inicio del curso (Tabla 1) se puede concluir que el $100 \%$ de los alumnos han trabajado en grupo con anterioridad al inicio del curso, tanto académica como lúdicamente (equipos deportivos), y por tanto han desarrollado experiencias previas acerca del trabajo colaborativo.

En cuanto a la cuestión 3, la mayor parte de los alumnos respondieron que esperaban aprender y obtener la máxima calificación posible del trabajo realizado

La tabla 3 muestra los resultados obtenidos de la cuestión 4, en la que se preguntaba a los alumnos sobre los aspectos que más valoraban al trabajar en equipo. El factor más importante para los alumnos es el compromiso de los miembros con el grupo, en segundo lugar el esfuerzo de los miembros del grupo, en tercer lugar la capacidad para generar ideas, $\mathrm{y}$ formas de trabajo.

Tabla 3. Aspectos valorados por los alumnos sobre los componentes del equipo

\begin{tabular}{lr}
\hline Commitment/ & $15,9 \%$ \\
Engagement & \\
Hard work & $13,4 \%$ \\
Innovation & $13,4 \%$ \\
Cooperation & $9,8 \%$ \\
\hline
\end{tabular}




\begin{tabular}{lc}
\hline Communication & $7,3 \%$ \\
skills & \\
Good environment & $6,1 \%$ \\
Efficiency & $4,9 \%$ \\
Learning & $3,7 \%$ \\
Organization & $3,7 \%$ \\
Right attitude/Mood & $3,7 \%$ \\
Discipline & $2,4 \%$ \\
Kindness & $2,4 \%$ \\
Loyalty/Trust & $2,4 \%$ \\
Autonomy & $2,4 \%$ \\
Initiative & $2,4 \%$ \\
Ambition & $1,2 \%$ \\
Competence & $1,2 \%$ \\
Open mind & $1,2 \%$ \\
Patience & $1,2 \%$ \\
Punctuality & $1,2 \%$ \\
\hline & Fuente: Elaboración propia \\
\hline
\end{tabular}

La última cuestión (pregunta 5) tenía por objeto hacer reflexionar a los alumnos sobre los aspectos a conocer de sus compañeros a fin de alcanzar sus objetivos personales (máxima calificación; aprender) a través del trabajo colaborativo.

Tras múltiples actividades grupales previas a la constitución de los equipos, se preguntó de forma individual a cada alumno, cuantos compañeros de clase habían conocido que fueran potenciales miembros de sus grupos. El resultado medio fue de 4 compañeros, siendo el mínimo 1, y el máximo 6.

Al finalizar el semestre, las coevaluaciones grupales del trabajo colaborativo desarrollado durante el curso dieron lugar a los siguientes resultados (Tabla 4). Nótese que todas las dimensiones del trabajo colaborativo fueron ponderadas por igual. Es relevante destacar que ningún equipo valoró con la mínima calificación (1) a su equipo en ninguna de las áreas del trabajo colaborativo.

Se observa de los resultados desprendidos de la columna (a) de la tabla 4 que las valoraciones grupales del trabajo colaborativo son altas, ya que la media se sitúa en el 8,05 sobre 10, mientras que la columna (b) muestra la evaluación realizada por el profesor del trabajo colaborativo de cada grupo a través de la presentación pública y el trabajo escrito (media 7,8). Se ha estimado el coeficiente de correlación de pearson para determinar el 
grado de relación lineal entre ambos indicadores, obteniéndose un valor negativo próximo al 0 , (-0,019), lo que denota una escasa, prácticamente nula relación entre ambos indicadores, y al mismo tiempo, negativa, es decir, que una mejor calificación otorgada por el profesor al trabajo escrito y oral de los alumnos, estaría relacionada con una peor valoración grupal.

Tabla 4. Resultados de la evaluación grupal del trabajo colaborativo (max 10)

\begin{tabular}{ccc}
\hline Equipo & $\begin{array}{c}\text { Puntuación rúbrica } \\
\text { (a) }\end{array}$ & $\begin{array}{c}\text { Puntuación profesor } \\
\text { (b) }\end{array}$ \\
\hline 1 & 8,57 & 8,55 \\
2 & 7,14 & 7,73 \\
3 & 7,50 & 6,88 \\
4 & 8,93 & 5,60 \\
5 & 7,50 & 8,61 \\
6 & 9,64 & 8,76 \\
7 & 7,50 & 8,20 \\
8 & 8,57 & 7,76 \\
9 & 6,79 & 7,94 \\
10 & 8,21 & 7,82 \\
11 & 8,21 & 8,70 \\
\hline
\end{tabular}

Fuente: Elaboración propia

\section{Conclusión}

Este trabajo supone ser un primer acercamiento a la coevaluación del trabajo colaborativo. Se ha creado un proceso para el desarrollo del trabajo colaborativo que ha dado lugar a una rúbrica inicial a través de la cual se fomenta la reflexión del alumno sobre sus objetivos individuales ante el grupo, sus valores ante el trabajo colaborativo. Con este sistema se fomenta la importancia del grupo como unidad de trabajo desde el instante en el que éste se constituye, ya que todos los individuos comparten objetivos.

Asimismo, del análisis realizado se desprende que las competencias de trabajo colaborativo actualmente medidas a través de la presentación oral y documento escrito pueden no son suficientes para evaluar las distintas dimensiones del trabajo colaborativo, por lo que es necesario tener en cuenta otros indicadores. Es por ello, que este trabajo se continuará analizando los resultados de la valoración que cada miembro del grupo hace del resto de componentes utilizando la misma rúbrica que en la evaluación grupal, si bien ésta es totalmente anónima. Así se podrá comparar la percepción consensuada del grupo, frente a 
la percepción que cada miembro del grupo tiene del resto de sus compañeros, y a su vez comparar los resultados académicos obtenidos a nivel individual y grupal. Se pretende dar respuesta a cuestiones relativas a si los alumnos más críticos con los compañeros son aquellos que obtienen mejores resultados académicos, como fruto de su carácter exigente al resto, o por el contrario los alumnos con mejores resultados académicos perciben las capacidades de sus compañeros más positivamente.

\section{Bibliografía}

BROWN, S., \& KNIGHT P. (2012). Assessing Learners in Higher Education. New York, Psychology Press.

CHEN, C.H. (2010). The implementation and evaluation of a mobile self- and peerassessment system. Computers \& Education 55 (1), 229 - 236.

FALCHIKOV, N. (1995). Peer feedback marking - Developing peer assessment. Innovations in Education and Training International 32, 175-187.

LOGAN, E. (2009). Self and peer assessment in action. Practitioner Research in Higher Education 3 (1), 29 - 35.

LUCAS, S. (2014). The Art of Public Speaking, 12th Edition, Mc Graw Hill Education.

RILEY, S. M. (1995). Peer responses in an ESL writing class: Student interaction and subsequent draft revision. Dissertation Abstracts International 56, 3031.

TOPPING, K. (1998). Peer Assessment Between Students in Colleges and Universities. Review of Educational Research 68(3), 249 - 276.

WILSON, S. (2002). "Comparing Peer, Self and Tutor Assessment in a course for University Teaching staff". Learning Communities and Assessment Cultures Conference. 